\title{
Measuring the Impact of Public Understandings of Risk from Urban and Industrial Development on Community Psychosocial Well-Being: a Mixed Methods Strategy
}

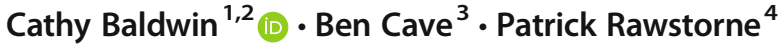

Received: 16 April 2019 / Accepted: 7 October 2019 / Published online: 26 November 2019

(C) The Author(s) 2019

\begin{abstract}
As the science of well-being moves towards an understanding of the influence of social experiences shared by many on individual and group-level well-being ('community well-being'), a new approach to measuring well-being is required. It needs to bridge the contextually-specific social experiences best uncovered by social research methods, and psychological diagnoses made using conventional psychometric scales and diagnostic interviews. We build on our previous work on a new psychosocial model of a major influence on contemporary community well-being, the process by which people form, maintain and change their understandings of risk from urban and industrial projects, and any subsequent effects on individual psychosocial well-being. We utilise this model, and propose a mixed qualitative and quantitative methodology to argue for; 1) the incorporation of the emic (subject's) perspective in the conceptual underpinnings of measurement scales; and 2) the synthesis of quantitative and qualitative assessments of well-being. This gives validity and contextual precision to scales which measure experiences of well-being that are geographically and socio-culturally-located. The resulting data offers both context of scale, and depth of insight. Additionally, our proposition combines theories and methods from psychology, social anthropology, sociology, social epidemiology, public health and community development. This evinces the importance of drawing on broad ranging perspectives to develop tools which capture the complex and multi-dimension nature of well-being - where psychological responses are shaped by collective social experiences.
\end{abstract}

Keywords Psychosocial $\cdot$ Public understand of risk $\cdot$ Community well-being $\cdot$ Mixed methodology $\cdot$ Engagement

Electronic supplementary material The online version of this article (https://doi.org/10.1007/s42413-01900041-x) contains supplementary material, which is available to authorized users.

Cathy Baldwin

cathy.baldwin0@gmail.com

Extended author information available on the last page of the article 


\section{Introduction}

Communities may develop understandings of the risks and benefits of urban and industrial developments in ways that impact upon their health and well-being. Industrial development will typically have effects during the construction stage which will be greatest for people closest to the development site. Sources of potential adverse health effects include exposure to hazardous biological, chemical and/or toxic materials used in industrial facilities; changes in land use, particularly spiritually important land; the relative distribution of economic benefits to residents and incoming workers; an increase in demand for direct and indirect sex work as a result of the construction workforce and the associated health risks for both sex workers and clients; and conflictual views between community members over the proposed development. There are different pathways by which community health and well-being can be affected by urban and industrial development during the construction, operation or decommissioning stages which can cause individuals in place-based, socio-residential communities to experience mainly psychosocial health and wellbeing effects, but also physical ones.

Public understanding of risk $^{1}$ is a determinant of community mental and physical health and well-being (Birley 2015). We previously published a new model (Baldwin and Rawstorne 2019) that captures the psychosocial and socio-cultural influences on the psychological process by which people form, develop and change dynamic understandings of risk that inform their attitude towards a development planning proposal, with reference to a developed body of literature on nuclear reactors and waste facilities. These are generally identified as conjuring up the highest levels of 'fear and dread' of all the public risks analysed (Taylor 2012, p. 9). Risks to health that are seen as 'uncontrollable and dreaded' are linked to higher (more adverse) health risk perceptions (Ferrer and Klein 2015, p. 86). The model could, though, be applicable to any development situation where risks are associated with potential health impacts ranging from low-level environmental annoyance to threats to life, e.g. possible exposure to chemicals, radiation, and pollution. We proposed that the model would be of interest to researchers and health impact assessment (HIA) practitioners exploring the links between understandings of potential risks, and community psychosocial health and well-being.

In this paper, we argue that our model provides fruitful territory for measuring the impacts of public understandings of risk on community psychosocial well-being. We introduce and discuss a new mixed qualitative and quantitative approach combining theories and tools from community development, social anthropology, sociology, psychology and social epidemiology. Individual mental health has historically been measured using established psychometric scales and diagnostic interviews. We argue that in capturing the dynamic influences on what are essentially risks to community or aggregated individual well-being, an interdisciplinary methodology and approach is needed that blends context of scale provided by psychometric testing with depth of

\footnotetext{
'In this paper, the term 'understanding of risk' is preferred to 'risk perception'. We acknowledge that the term 'risk perception' is more commonly used in the literature. We use the term 'understanding' in preference to 'perception'. An understanding of a topic or an issue is something that can develop and that can be debated and shared. The term 'perception' can imply views that do not align with scientific analysis are given less credence.
} 
insight into the influences on well-being that communities self-identify through their participation in exploratory qualitative workshops.

We also describe how the self-reported data generated by these methodological and engagement tools can be categorised as low, medium or high levels of risk understandings. This would guide well-being advocates, local authorities, urban planners, HIA assessors working with developers in reviewing all the evidence of potential health and well-being effects that some individuals within communities may experience, and ranking their magnitude, duration, frequency and geographic influence, and other sectors.

\section{Why Use a Mixed Methods Approach to Measure Community Well-Being?}

Clinical psychiatry and psychology use quantitative psychometric scales to rate individuals' mental health and wellbeing statuses, in addition to the clinical interview for diagnosing mental ill health and ill-being. They employ diagnostic criteria set by experts from two international standards, the World Health Organization (WHO)'s International Classification of Disease Version 10 (ICD-10) and the American Psychiatric Association's Diagnostic Statistical Manual Version 5 (DSM-V) ${ }^{2}$ that are adapted for different measurement instruments. In the early 2000s, there was a growth of interest in 'positive' mental health (see Huppert and Wittington 2004; World Health Organization 2004) at individual and population-levels. More localised, purpose-built well-being scales emerged such as the Warwick-Edinburgh Mental Well-being Scale (Tennant et al. 2007). This scale has some grounding in communities' own perspectives on well-being, as community groups and mental health service users participated in focus groups around England and Scotland to discuss their personal concepts of positive mental health in relation to a previous scale. These were used to inform the final selection of scale items by a group of experts.

Positive mental health was underpinned by a shift from the construction of the mentally unwell person as a powerless individual unable to control the symptoms of mental illness and ill-being to the individual's potential to achieve a positive state of subjective mental well-being. Mental health research has shifted again towards an emphasis on people developing psychological resilience, flexibility and accepting the full range of emotions, rather than maintaining constantly positive feelings. However, 'positive mental health' usefully combined the traditional hedonic perspective on wellbeing (Ryan and Deci 2001) - a more passive subjective experience of positive emotions and life satisfaction (Waterman, 1993), with the eudaimonic perspective encompassing human effort in achieving a positive state of being by 'being holistically engaged, being challenged and exerting effort' (Anderson and Baldwin 2017, p. 316) leading to psychological functioning and self-realisation (Tennant et al. 2007, p.3).

Eudaimonia's emphasis on the individual engaging outwards chimes well with the concept of social health and well-being, and recent interest in extending measurements of individual well-being status to the group- or community-level. The literature on social health and well-being (e.g. Waterman 1993; Keyes et al. 1998; 2008) recognises it as a

\footnotetext{
${ }^{2}$ See https://www.who.int/classifications/icd/en/GRNBOOK.pdf and https://www.psychiatry. org/psychiatrists/practice/dsm
} 
distinct phenomenon, e.g. Keyes et al. (2008, p. 100; WHO Constitution 1948/2006) differentiated from individual psychological and emotional well-being. Whilst there is no widely accepted common definition of 'social health' (Larson 1993, p. 287), it can be conceived of as the health of society and factors such as the distribution of wealth (MacDowell and Newell 1987), or the influence of social phenomena on individuals. Social well-being is inherently rooted in the experiences an individual has in the wider social environment or societal context. Such experiences, by the very meaning of 'social', involve interaction with others, and by their nature, may be shared. It is argued elsewhere that 'a well-lived life includes the quest for positive social lives, involving meaningful interaction with family, community and wider society' (Anderson and Baldwin 2017: p. 317). Therefore, attempts to measure social well-being must capture the influence of social factors that may be experienced and felt by many, i.e. at the community level.

Fine formulation of the components of social well-being constructs already exist (see Keyes 1998). However, from a conceptual and methodological standpoint, we contend that psychosocial approaches (see Martikainen et al. 2002) are among the best for the study of well-being in social communities. They combine theories of individuals' internal psychological processing with insights from the social sciences (i.e. sociology, anthropology, social psychology) that attempt to explain the social influences on the human brain and behaviour.

Social epidemiology (see Berkman and Kawachi 2000), an evolving field, is also useful to the science of community well-being. It employs a public health socio-ecological model of the 'determinants' of health and well-being, i.e. the factors which that determine individuals' health and well-being status. In a social sense, it explores causal pathways between social factors ('social determinants'; Dahlgren and Whitehead 1991; Wilkinson and Marmot 2003) that influence health and well-being statuses, and the health effects of this influence. It allows us to identify the symptomology of poor mental well-being at the social or group level. We employed this approach to measure subjective well-being in individuals in geographically-delineated neighbourhoods with overlapping social communities in England (Baldwin et al. under review), i.e. the influence of social factors that affect many people's well-being. The next step will be to create measures that assess wellbeing among whole groups without aggregating individual measures. One conceptual and ethical quandary that returns us to the present paper concerns the democratisation of measurement scales. If, as with traditional diagnostic criteria, experts set the criteria for psychosocial well-being among a particular population, there is a risk that researchers will fail to capture the social factors and experiences that influence social and mental wellbeing among certain people. We have not observed well-being researchers attempting the same deep immersion in the social experiences of local populations as social anthropologists undertaking ethnographic research.

Anthropologists use the distinction between emic (from the study subject's point of view) and etic (from the analysts' point of view) to position their analyses as close to their subjects' viewpoint as possible (see for example, Harris 1979). This is a helpful benchmark for community psychosocial well-being scales and methods of constructing them. Although quantitative well-being researchers routinely apply validity tests (e.g. face and content validity) to their instruments using techniques such as cognitive interviewing, we advocate a fully mixed methods approach to the construction of models and scales measuring well-being and its influences. We make the case for including the emic perspective in scale development. 
We outline a mixed methodology for studying a powerful influence on communitylevel psychosocial well-being: the public understanding of potential risks from infrastructure and energy projects. It combines the qualitative participatory method of assetbased community development workshops (Glasgow Centre for Population Health 2011) to assist in the development of a survey questionnaire, to independently complement the quantitative data generated, and enable data triangulation. Our methodology is underpinned by our previous work (2018) on the psychosocial model capturing the social and cultural influences on individuals' psychological processing when they develop understandings of specific risks to their personal health and well-being. These understandings may result in the evolution of an attitude towards a development proposal that causes the person to experience symptoms of poor psychosocial wellbeing. These may affect multiple individuals in the same community. We regard this paper as a cry to well-being researchers to adopt mixed methods.

\section{Methodology}

See Baldwin and Rawstorne (2019) for a description of the methodology behind the psychosocial model. The methodology proposed here has four steps: 1) a public information campaign; 2) asset-based community development workshops; 3) a questionnaire survey, and 4) community feedback and refinement workshops. We describe each in detail and how it was selected, how the methods are combined and applied, inform each other, and allow for data triangulation after data collection in the main body of the text. A basic version of the mixed methodology described here was developed by Baldwin and Rawstorne for use in a UK public health project which remains anonymous due to confidentiality clauses. The asset-based community development $(\mathrm{ABCD})$ workshop schedule was devised using $\mathrm{ABCD}$ theory and practice (Brown 2015; Glasgow Centre for Population Health 2011) and the model of the 'social determinants of health', e.g. where communities discuss those seen as 'community assets' that may be changed by development plans with potential health and well-being effects. The workshop format was successfully delivered with positive feedback via written evaluation forms completed by the community in the previous health project. This design was adapted further for this and our previous paper to include a preliminary list of the social and cultural influences on understandings of risk - identified from an academic literature review (see methodology in Baldwin and Rawstorne 2019) - from which the community select and refine to create a final list in the workshop.

The approach to mixed methods proposed here involves what Creswell et al. (2003) may describe as sequential design. It involves sequential explanatory aspects in which the research literature is helping to inform both the design of the qualitative workshop questions as well as the constructs to be measured in the quantitative questionnaire. It also involves sequential exploratory aspects in which the qualitative data from the workshops informs some of the questions in the questionnaire as well as the overall interpretation of the project findings. The qualitative to quantitative link also helps to examine whether issues that were pertinent at a qualitative level apply to a broader cross-section of the potentially affected community. There is no priority given to either the quantitative or qualitative approaches. Rather, each approach is seen as informing the other. 
For this paper, author Baldwin drafted a simple sociological version of the questionnaire including the 5-point Likert scale utilised based on a version used in the public health project. The 5-point scale was chosen over 3- or 7-point scales, as it provided sufficient answer options for respondents to indicate the different strengths of their agreement or disagree, with each statement in the questionnaire. When used in the public health project, the survey provided enough data but not too much to overwhelm data analysts. Data collection was achieved by a research company using telephone interviewers in 3 weeks, and analysis was performed by a four-person team (including Baldwin and Cave) over a period of 2.5 months (not full time). For the present paper, Rawstorne further developed the questionnaire in consultation with Baldwin by drawing on existing psychological scales used in research settings, and amalgamating them into, and adapting the existing instrument. This paper is accompanied by an Appendix which includes a complete workshop interview schedule, Workshop Schedule for Workshops 1 and 2 (pp.3-9), and spatially compressed Psychosocial Environmental Questionnaire (pp.10-13), to which the remainder of this article refers.

\section{The Psychosocial Model}

Here we offer a summary of the model. A full explanation is available in Baldwin and Rawstorne (2019).

\section{Aim of the Model}

The key theoretical aim of the model is to link the social, visceral and emotional experiences a person has in developing an understanding of risk from a proposed development with the underlying psychological processes, and ultimately, with any possible mental and physical health and well-being outcomes (the biopsychosocial spectrum). The pragmatic purpose of the model is to provide a conceptual framework that can be tested empirically and which will help to predict and explain the processes and factors involved in individuals and communities developing an understanding of risk, which in turn, influences the development of an attitude towards the development, and emergence of any associated health and psychosocial well-being effects. Therefore, the aims of the model are for it to be both predictive and explanatory. It is a causal model specifying causal pathways between social and cultural factors and health and psychosocial well-being outcomes. It is also an explanatory model in purporting how understandings of risk are shaped.

\section{How Does the Model Work?}

The model illustrates the psychological process through which a person forms, develops and changes their understandings of risk. It is a multi-directional causal model that recognises the dynamic interplay between social, psychological and biological factors in the ways in which understandings of risk affect people's health and wellbeing. When a person first learns about a potential risk to their health, e.g. a proposed nuclear power station, their response is shaped by two factors internal to the human brain: 1) personality traits/dispositions (optimism/pessimism), and 2) their immediate 
psychological response to the specific risk, based on a combination of deliberative, affective and experiential psychological processes (see Baldwin and Rawstorne 2019). ${ }^{3}$ These two internal factors mediate understandings of risk, which are then moderated by three categories of social and personal factors 1) personal experience, 2) information, and 3) contextual factors, e.g. specific socio-cultural, demographic, and temporal factors, and additional psychosocial or cognitive/emotional influences, e.g. affect (emotion), social attitudes, and personal or social identity formation and/or reinforcement in response to social experience. Through this whole psychosocial process, a person's understanding of risk evolves.

In the model, risk understandings are cognitions that mediate between: 1) all the information a person receives about the development project - e.g. from a range of sources in the social environment, i.e. media and gossip [external information] and the person's own thoughts and feelings [internal information]); and 2) their personal attitudes about the development. Their attitude/s, in turn, influence any subsequent health and well-being effects (psychological and physical) such as the development of symptoms of psychosocial ill health and ill-being.

However, people's understandings of risk are not static but are dynamic and changing (Pidgeon et al. 2008). They are local-context specific (Hamilton 1985, p. 480; Greene et al. 2014; Marcon et al. 2015, p. 276), vary across heterogeneous communities and may be sensitive to temporal shifts (e.g. as new information about the source of risk emerges over weeks or months). Thus, the issues that may be most relevant to shaping understandings of risk in a given industrial development when a planning application is being prepared may change over time, as may the levels of risk understandings (i.e. low, medium, high), and an individual's attitude towards the development. Our proposed methodology allows well-being researchers to collect evidence of potential poor psychosocial well-being that may be associated with development proposals. By engaging in dialogues with developers and planning authorities, well-being advocates may push for changes to the proposal if communities understand the risk to be high. Such changes may lead to improvements in the symptoms of poor psychosocial well-being across communities, and consequently catalyse changes in the strength of public understandings of risk, e.g. high to medium or low.

Figure 1 depicts the psychosocial model. It is used to underpin the mixed methodology unveiled next. All of the factors in rectangular boxes in the model represent variables that could be measured using a questionnaire while experiential responses contained in the only oblong shape is a latent variable that is inferred statistically from the measurement of both deliberative and affective responses. The model is causal in that it specifies the proposed directions of influence of various factors on psychosocial health and well-being and physiological health. It is expected to predict as well as explain the factors underpinning understandings of risk about a development project.

\footnotetext{
${ }^{3}$ Deliberative responses are 'rational', 'systematic, logical and rule-based' (Ferrer and Klein 2015, p. 86), where a person relies on 'reason-based strategies' to judge the likelihood of a negative outcome from a risk. Affective responses are the emotions associated with an understanding of risk. Experiential responses are the result of a rapid psychological negotiation between deliberative and affective responses, refer to the content of the understanding of risk, and are 'consciously accessible' to the individual (Ferrer and Klein 2015, p. 86), e.g. 'My gut is telling me that $\mathrm{x}$ will result in $\mathrm{x} . .$. '. As such, experiential responses are depicted in the conceptual model as being derived from both deliberative and affective responses.
} 


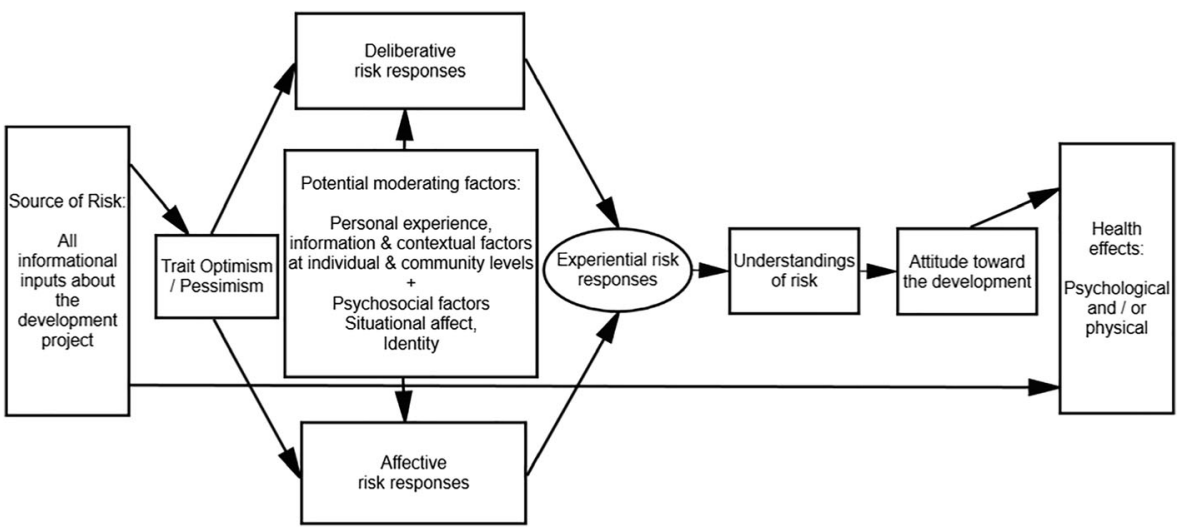

Fig. 1 Psychosocial conceptual model of formation of public understandings of health risk

\section{The Impact of Public Understandings of Risk on Psychosocial Well-Being}

We argued previously (Baldwin and Rawstorne 2019) that living with an understanding of the risk of adverse health effects may, via individual attitudes towards a development, mediate psychosocial and physiological health and well-being effects. See our first paper for a full review of potential psychosocial well-being effects.

\section{Outline of the Mixed Methodology}

\section{Overview}

Our chosen approach, asset-based community development (ABCD), is a theory, methodology and approach to public health promotion and community development that underpins our methodology. It was selected due to Baldwin's experience of applying it with positive results in public health promotion projects. ABCD's aim is the collective organisation and empowerment of people through a process whereby they identify both tangible and intangible resources or "assets" (Brown et al. 2015, p. 126) at their disposal (Laverack 2001, p. 140), and mobilise to address common 'problems or goals' (Nutbeam and Harris 2004, p. 31; Laverack 2001, p. 139) in health and well-being, and 'develop and implement strategies' (Nutbeam and Harris 2004, p. 31) and actions to combat them. Outside organisations (Laverack 2001, p. 140) specialists often partner with 'communities, individuals, community groups, workers and leaders' (Nutbeam and Harris 2004, p. 32) to plan and implement interventions within the group social context over a long time-frame.

Developing an understanding of a risk to health, well-being and quality of life in one's local area and socio-residential community is inherently disempowering due to the lack of control and powerlessness that individuals may feel, and any consequential symptoms of poor psychosocial well-being. The objective of employing ABCD as an underpinning theory and a practical approach to collecting qualitative and quantitative data on public understandings of risk from potentially affected people is, therefore, to give respondents feelings of control, empowerment and ownership over the process. 
These feelings act as a protective buffer against any potential incidental effects on psychosocial well-being (i.e. anxiety) that may arise from confronting the uncertainty that participants experience, in not knowing whether the proposed development will have adverse health and well-being effects, a cause of stress.

\section{When would the Methodology be Useful?}

Our methodology could be implemented to collect data at the midpoint in time between the announcement of the development proposal and the final decision about whether it will proceed. Potentially affected community members are likely to have absorbed public information about the proposal, and be forming understandings of risk that are subjectively shaped by external influences in their immediate geographical and sociocultural environment. Before proceeding with data collection, ethical approval should be sought from a health authority, as our instruments ask brief questions pertaining to indicators of respondents' current personal psychosocial well-being. This is so that the outcome measures in our proposed questionnaire show the possible effects on community psychosocial well-being, for the benefit of well-being advocates.

\section{The Four Methodological Steps}

Our methodology could be implemented to collect data at the midpoint in time between the announcement of the development proposal and the final decision about whether it will proceed. The steps involved in this research activity are summarised in Fig. 2 and provided in more detail here.

\section{Step 1: Conduct a Public Information Campaign}

The methodology entails sampling participants from the affected geographical area from where data will be collected. However, before data collection, the first step is a public information campaign within the potentially affected area. Well-being researchers or HIA practitioners should inform residents that they may be invited to attend workshops and/or complete the survey, explain the sensitive nature of the inquiry, and that participation is voluntary. Locally appropriate communications channels should be selected for the dissemination of these messages, e.g. social media such as Facebook and Twitter, leaflets through doors, posters in public places, community radio broadcasts, megaphone announcements, adverts in newspapers, messages relayed via word of mouth through networks, community ambassadors etc.

\section{Step 2: Hold Two Exploratory workshops (see Appendix p.3-10)}

\section{Qualitative Methods: Exploratory Workshops}

The second step is two exploratory workshops to cultivate initial trust and understanding between the researchers, professional stakeholders, and residents before researchers can 


\section{Step 1: Conduct a public information campaign}

- To inform residents about the inquiry and its sensitive nature; that they may be invited to attend workshops or complete a questionnaire, and that participation is voluntary.

\section{Step 2: Hold two exploratory workshops (Appendix pp.3-9)}

- Workshop1 - Stakeholder workshop

- A multi-stakeholder workshop is held first to reduce the list of potential external social and cultural influences on understandings of risk to those which are locally relevant (Appendix: Table1)

- Key professional stakeholders and a diverse selection of pro-active and community members who live among and work closely with affected communities and are likely to have a good understanding of local concerns, should be invited to the first workshop.

- Workshop 2-Community workshop

- A second workshop or series of workshops with various sub-groups are held for people to share knowledge of their geographic area and sociocultural environment that may affect their understandings of risk.

- In the second part of the workshop, the refined list of external social and cultural influences (Appendix: Table 1) can be further narrowed down to those which the community holds are contextually relevant. These are then are inserted into the questionnaire (Appendix pp.10--13).

- Finally, the researchers carefully moderate a whole workshop discussion, which identifies possible solutions to the main potential challenges

\section{Step 3: Administer the survey and analyse the data (Appendix p.10-13)}

- The questionnaire measures the different influences on the formation of public understandings of risk; the development of an attitude towards the development; and any symptoms of psychosocial ill-being, indicative of the impact of the development on psychosocial well-being.

- The questionnaire is based on the psychosocial model shown in Figure 1 and is developed from the generic questions provided in the Appendix (Table 1, pp.3-9) as well as the addition of the final list of external influences decided upon by participants in Community Workshop 2.

- Questionnaire data would be analysed to test the strength of the relationships between variables that are purported to underpin Understandings of risk and whether such understandings are associated with attitudes and indicators of psychological health effects. Note: the questionnaire does not attempt to measure indicators of physical health.

\section{Step 4: Hold a feedback and refinement workshop}

- Survey results are shared with community members at a final workshop, with the support of professional stakeholders. The community reviews the results and provides feedback (e.g. on disagreements they have, or exceptions, variations or anomalies that they are aware of).

- If mitigation measures or public communications have been developed using qualitative data from the first workshop, these can be reviewed and validated.

Fig. 2 A summary of the four steps involved in collecting relevant data 
ask the latter two groups sensitive questions using the survey. These workshops are held before the survey commences, which itself asks the most challenging and personal questions in our mixed method data collection exercise. This is to prevent adverse incidental psychosocial health and well-being effects from occurring as a result of the survey's inquiry by using positive social relations cultivated during workshops as a buffer to cushion any after-effects of thinking and talking about challenging topics. It is also to gather reliable qualitative data on the public understanding of risk; narrow down a pre-existing list of potential external influences on public understandings to those which are locally pertinent - as compiled from our literature review (see Baldwin and Rawstorne 2019); and as regards one of those influences - local socio-cultural factors that may impact on residents' understandings within affected geographic areas - to identify relevant ones.

\section{Workshop 1: Stakeholder Workshop}

A multi-stakeholder workshop is held first to reduce the list of external social and cultural influences on understandings of risk (see Appendix: Workshop Schedule for Workshops 1 and 2, pp.3-9) identified down to those which are locally relevant from three broad categories: 1) previous personal experiences of the urban or industrial development being proposed, 2) available sources of information on it and its potential health effects; and 3) local contextual factors that will shape understandings.

The literature review in our earlier paper (2019) identified influences from these three categories based on academic studies addressing empirical contexts in the UK, USA, Canada, Australia, Russia, Ukraine, Portugal, Switzerland, Germany, Italy, Japan, Taiwan etc. For the present paper, this list was refined to include only influences which easily translated into measurement variable (which are used in the second community workshop described below). These are listed in the Appendix under Workshop Schedule for Workshops 1 and 2 (pp.3-9). This schedule includes a description of each influence in the first column. In the second column, respondents are asked questions (listed) about how each influence affects an individual's understanding of risk. In the third column, a factor-specific 4-point Likert scale, or in one case, a binary yes/no answer option, is offered for measuring the extent of each influence. In the first workshop, a Stakeholder Workshop, the list of external influences is discussed by participants only in order to refine it to those which are locally relevant.

The influences that we have included in Table 1: Workshop Schedule for Workshops 1 and 2 (Appendix: pp.3-9) can be substituted by other factors from any national, cultural and community contexts. The psychosocial model is designed to be applicable in cross-cultural contexts, and the external influences can be augmented by any found in locally contextualised research studies. Table 1 (Appendix) is divided into two lists, 1 and 2. List 1 contains those influences on understandings of risk (in the first column) in the context of a new build development (Appendix: p.3-9). List 2 (first column) contains influences that apply where an existing development is being upgraded or rebuilt (Appendix: pp.7-9). Depending on whether a proposed development is a new build or an upgrade of an existing facility, any workshop should use either List 1 or 2. In both Lists 1 and 2, the influences in Category 1: Personal experience, are subdivided into those which affect understandings of risk before and after the public announcement of the proposal. 
Key professional stakeholders who live among and work closely with affected communities, and are likely to have a good understanding of local concerns, should be invited to the Workshop 1: Stakeholder Workshop. These may include council community relations or development officers, and NGO advocates. A diverse selection of pro-active and resilient community members who, by choice, advocate on behalf of more vulnerable individuals and are less likely to be adversely emotionally affected, should also attend. The workshop comprises moderated group discussions. In the first discussion, participants reduce or substitute items from the Workshop Schedule for Workshops 1 and 2 (Appendix: pp.3-9). For example, in List 1, Category 1, sub-category b), influence No. 6): 'The perceived contribution of the proposed development to the social life of the community' may not apply if the proposal for a new facility has not provided social opportunities for a community as part of the preparation of a planning application. From List 2, Category 2), influence No. 2): 'The extent to which risk communications are perceived to be clear' may not be relevant if the facility is long-established, and there have not been recent risk communications. In the second discussion, socio-cultural factors that may colour residents' understanding of risk from the development (cf. Hanna et al. 2016) are identified for inclusion in the schedule under the item: Category 3, influence No. 1.

\section{Workshop 2: Community Workshop/s}

A second workshop, using the $\mathrm{ABCD}$ technique (or several workshops to reach important population sub-groups, e.g. by age, gender, socio-economic status, disability, ethnicity and race, parental status) should be scheduled. The researchers should sample community members who are most likely to be adversely affected by a high understanding of risk and invite them to participate with support from experienced facilitators. The size of the workshop depends on local resources and constraints. Fifty participants per workshop is a reasonable number. The workshop/s should be held at times, in places and with access supports (e.g. creches, transport, translation) that meet target participants' needs. The workshop aims to give community members an opportunity to: 1) share knowledge of their geographic area and socio-cultural environment that may affect their understandings of risk; and 2) to discuss and share views on the finalised list of external influences (see Appendix). These discussions should be recorded and transcribed to generate qualitative data. It is acknowledged that all 'communities' are internally diverse, and that unequal power relations between individuals in group discussions may risk allowing dominant voices to most influence the direction of the discussion (see Parry and Wright 2003 for further discussion). Skilled moderation that draws out quieter voices and garners equal airtime for all perspectives to be heard will be required. No participatory process is without flaws and risks, but skilled moderation may mitigate this risk.

In the first part of the workshop, groups of participants identify the strengths, assets and weakness ('likes'/'dislikes') - social, bio/physical, economic, cultural, political, or otherwise - in the affected geographic area. They mark where, when, and among whom these 'likes'/ 'dislikes' occur on maps of the locality using creative tools such as coloured pens and string, sticky notes, stars and dots. When identifying and reflecting on the 'likes' and what could be protected, or improved (the 'dislikes'), participants become agenda setters and area guides. Sharing knowledge and collective reflection can bolster participants' self-esteem, prove empowering and build the community's collective capacity. A note-taker observes and records each group discussion as another 
source of qualitative data. A group member summarises the key points back to all participants, and researchers plot these on a board or large sheet of paper.

In the second part of the workshop, the refined list of external moderating factors in Workshop Schedule for Community Workshops 1 and 2 can be further narrowed down to those which the community holds are contextually relevant. Experienced moderators ask for each groups' views on these influences. By this stage, participants are sufficiently engaged and familiar with the working format to respond directly to these specific factors. The researchers can use the questions proposed in the Workshop Schedule to ask participants how much they believe each factor is important in shaping their understandings of risk about the development proposal, and then use the Likert scales to record the measurement (see Psychosocial Environmental Questionnaire, Appendix: pp.10-13). The refined list of external influences is inserted into the questionnaire, so that the relative importance of these factors is tested on a larger scale, and moderation effects can be tested during statistical analysis of the psychosocial model (see question 10 in the Appendix: p.13). This is discussed in the section of this paper entitled 'Quantitative Data Analysis' (below).

Then, rather than asking direct questions about understanding of risk to community health and well-being, the groups critically address their hopes and possible challenges for it in light of the development proposal, and share their thoughts or feelings in a safe situation. Moderators can steer each discussion to balance health challenges with hopes for positive outcomes and benefits. For example, fears for poor air quality or environmental pollution, with hopes for investment in healthcare infrastructure and improved social cohesion due to new job prospects for local people. This section concludes with a moderated summary discussion where each group reports back to all participants on key points.

Finally, the researchers carefully moderate a whole workshop discussion, which identifies possible solutions to the main potential challenges, by drawing on the local strengths/assets mapped out earlier, which are displayed in a list or on a map. Participants propose solutions or mitigation or management strategies, and even alternative development proposals. At the end, they complete feedback evaluation forms, and are briefed that they may be contacted and asked to complete the questionnaire. The researchers write up their workshop notes, analysing and incorporating the qualitative data from the annotated maps, evaluation forms and group discussion transcripts. Thematic analyses can be used to organise and analyse these data by each determinant of health and well-being that participants think may be changed if the proposed development proceeds, resulting in risks to health and well-being. Researchers circulate a draft report to participants who provide feedback at community meetings, and through local communications channels.

\section{Step 3: Administer the Survey and Conduct Quantitative Data Analysis (see Appendix pp.10-13)}

\section{Quantitative Methods: the Psychosocial Environmental (Survey) Questionnaire}

Following the second workshop, the third methodological step is the administration of the survey questionnaire. It measures the different influences on the formation of public understandings of risk, the development of an attitude towards the 
development, and any symptoms of psychosocial ill-being. These symptoms indicate the extent to which the understanding of risk, via attitude towards the development, is impacting on psychosocial well-being (e.g. the absence of such symptoms). The questionnaire is either interviewer-administered by researchers or selfcompleted by participants. The latter is only possible for samples of literate people. Researchers select a larger sample of participants to complete the questionnaire than whom attended the workshops - see the section on Sampling and Data Collection below. They employ the most locally appropriate randomised method, e.g. telephone calls, paper questionnaires, or household visits. The questionnaire instrument itself is comprised of generic questions relevant to most types of development. Each question is underpinned by a variable used in the psychosocial model shown in Fig. 1. We provide items/questions to measure each variable in the spatially compressed Psychosocial Environmental Questionnaire in the Appendix (pp.10-13). We describe the rationale for, and function of each question here. The question numbers correspond with the questions in the Appendix.

\section{Warming Up Questions}

\section{Question 1: Likes/dislikes}

The questionnaire begins by asking respondents to nominate their likes/dislikes about their geographic area in a free-text question. Researchers can analyse and categorise them into local assets/weaknesses so that they can be retained and drawn upon to propose measures to developers, planning and health authorities to mitigate any potential negative effects on psychosocial well-being.

\section{Question 2: Gentle Psychosocial Moderating Influences}

The questionnaire then asks about gentle psychosocial moderating influences, e.g. whether respondents have a clear cognitive connection with the local area ('sense of place'), whether they feel attached to the local area ('place attachment'), if they have a strong local identity. This allows respondents to warm up, relax, and respond on topics which empower them by soliciting local knowledge. Each question is underpinned by a well-defined psychosocial concept and asked using a closed statement format. See Baldwin (2015) for definitions of concepts describing the ways that people develop connections and emotional attachments to places and communities.

These items would be measured on a Likert scale from 1 Strongly disagree; 2 Disagree; 3 Neither disagree or agree; 4 Agree; 5 Strongly agree, and when more than one item is being used to compute a construct scale score, as for 'sense of place' a mean score would be derived from respondents' answers to these items. Opt-out answers such as 'Don't know' and 'Don't want to answer', which ought not to be included in the calculation of a construct mean score, can also be added to the right of the scale on any self-completion survey format. These may reveal issues that are sources of confusion or which generate complex opinions that cannot be easily categorised, which is in itself revealing and informative. 


\section{Question 3: Situational Affect}

The second type of external moderating variable, situational affect (emotion), should be measured using an established psychological scale of markers of positive affect. We used Huppert and So's (2013) flourishing or mental well-being scale. There are ten items in the scale which were sourced from the larger European Social Survey (ESS) some of which we have modified. The ten items ought to all be measured on the same 5-point Likert scale, as for question 2 and all other questions employing a Likert scale answer format in the questionnaire. One item is reverse scored - see Question 3 in the Appendix - and then all items are combined and a mean score produced. There are no cut-off scores as this is not a diagnostic instrument but rather an indicator of positive affect. The construct is analysed as a continuous variable with higher mean scores being indicative of higher positive affect.

\section{Public Understanding of Risk Questions}

The more direct questions on public understanding of risk are located in the middle of the questionnaire after the gentler questions about local knowledge. By now, respondents are familiar with the survey format, thus it should feel easier to respond. Questions are phrased in intentionally gentle and respectful language to mitigate any adverse effects of discussing the issue.

\section{Source of Risk}

Although it is included in the model, source of risk is not measured and tested in the questionnaire as residents will have been exposed to a range of information from various sources about the proposed development, some of which will be more credible than others. The position of this variable in the model illustrates that the information to which people are exposed is varied and outside the control of any single agency.

\section{Question 4: Trait Optimism / Pessimism}

The personality dimension of optimism-pessimism is positioned in the model as some people have a tendency to interpret information optimistically, while others have a pessimistic slant, and a majority lie somewhere along the continuum between strong optimism and pessimism. Compared with people who are more pessimistic, optimists are likely to perceive fewer risks from a development and experience less anxiety (Lima 2004; Jacquet and Stedman 2014; Marcon 2015).

It is not necessary for researchers to measure the optimism / pessimism dimension unless they intend to test or conduct research on the psychosocial conceptual model. In which case, measuring optimism / pessimism is important for testing the purported relationships in the model, including any moderation effects between optimism / pessimism and deliberative and affective risk responses. If they are not testing the model, then measuring optimism / pessimism is unnecessary and arguably unethical. There will always be a range of personality types in any community, and personality is a relatively stable trait across the lifespan and therefore offers no point of intervention even if there was a justifiable reason for attempting to do so, which there is clearly not. 
Most important is that people approach situations differently and the optimism / pessimism dimension may underpin a range of understandings of risk in response to the same source of risk. For those intent on measuring the optimism / pessimism dimension, we suggest the 10-item psychometrically reliable and valid Life Orientation Test - Revised (LOT-R) by Scheier et al. (1994). Scoring is continuous and there are no cut-off or threshold levels to indicate optimism or pessimism. The 5-point Likert scale is used to record answers, as before.

\section{Question 5: Deliberative Risk Responses}

It is important to measure deliberative understandings of risk as these reflect conscious and deliberate, rational thought processes about perceived risk. Items to measure the domain of deliberative risk understandings must include a consideration of the risk or lack of risk. The 5-point Likert scale is used to measure answers as before and a mean score would be derived from respondents' answers to these items. The items we proposed were developed or derived primarily from Ferrer and Klein (2015), Higginbotham et al. (2007), and Lima and Marques (2005). Four items - 2, 4, 6, and 8 - are reverse scored - see Question 5 in the Appendix - and then all items are combined and a mean score produced to form a scale ranging from 1 to 5 , with a higher score representing a more positive deliberative risk sentiment.

\section{Question 6: Affective Risk Responses}

It is also important to measure affective risk responses as these will reflect how individuals in the community are feeling about the proposed development. This domain potentially taps into both conscious and unconscious processing (Oatley and JohnsonLaird 2013). According to Oatley and Johnson-Laird, cognitive appraisals (evaluations; judgements of value) precede and determine emotional responses. When such cognitive appraisals are outside conscious awareness, as they sometimes are, individuals may at times be unclear about the origins of their emotional responses and attribute the source of their affect incorrectly (Schwarz and Clore 1983). In the psychology literature, it is accepted theoretically and has been demonstrated empirically that emotional states sometimes precede and influence cognitions (Zajonc 1980). And in response to an emotional reaction, people will try and make cognitive sense of their experience (e.g. Festinger 1957; Schwarz and Clore 1983). The relevance here is that the measurement of affective understandings of risk ought not to be interpreted as an indicator of the extent to which the proposed development has caused a person to have an affective response. Affective responses are not always created from a stimulus alone (i.e. an industrial development), but from a combination of a stimulus and the way a person appraises (interprets and evaluates) that stimulus as being positive or negative (Lazarus 1999). What people believe to be important is what ultimately matters in developing understandings of risk, which then forms an attitude, and may subsequently lead to psychological health effects.

It is also important to note that the experience of unpleasant emotions in response to learning about an industrial development may be a normal and healthy response. By acknowledging and making room for emotional experiences, people are in a much stronger position to effectively process those emotions and the thoughts that go with 
them. And since emotional experiences are dynamic and changing, an unpleasant emotional response experienced early on in the planning for a development may later develop into more positive sentiment as more information comes to light. The opposite may also be true: that initial positive sentiment about the development may develop into negative sentiment. Such scenarios highlight how important it is for researchers to realise that the psychosocial model captures only a limited snapshot of what may be occurring cognitively and emotionally at a given point in time which may look different at another point. As such, the psychosocial model may be used to track changes in community sentiment over time and/or in response to interventions by administering the questionnaire on more than one occasion.

In developing items to measure this domain, one must include an emotion linked with an aspect of risk; the type of emotion indicating whether their feelings are negative or positive. Items would be measured on a Likert scale as before, and a mean score would be derived from respondents' answers to these items. We have devised original items in the questionnaire with examples of emotions written into the questions (see Appendix - Question 6). Three items - 3, 4, and 5- are reverse scored - see Question 6 in the Appendix - and then all items are combined and a mean score produced to form a scale ranging from 1 to 5 , with a higher score representing a more positive affective risk response.

\section{Experiential Understandings of Risk}

Experiential risk perceptions are conceptualised as a cognitive negotiation between deliberative and affective risk perceptions. They are not measured directly but are inferred from the measurement of deliberative and affective risk perceptions. ${ }^{4}$

\section{Question 7: Understandings of Risk}

This domain conceptually represents a person's assessment of all the information they have processed about the proposed development to arrive at an overall view about whether the risk outweigh the benefits or not. This domain is measured using a semantic differential scale because it is easy for people to answer and is known to tap into connotative meanings which may otherwise be too complex to articulate. While use of this scale will not provide information about why a person answered in a particular way, that level of detail will be available through participants' answers to the deliberative and affective risk response domains.

A mean score would be derived from the sum of respondents' answers on the semantic differential scale - see section on 'Analysis of the data' for further information on scoring construct scales. As an alternative to using the continuous variable and computing a mean score, an ordinal categorical variable could be created by dividing the sample into three categories based on the frequency distribution of the variable: the bottom third (understandings of risk as low), the middle third (understandings of risk as moderate) and the highest third (understandings of risk as high). Further analyses would then be based on the new categorical variable. Choice of the continuous versus

\footnotetext{
${ }^{4}$ This is done through statistical techniques using structural equation modelling where experiential understandings of risk would be considered a latent variable, or through path analysis using regression methods.
} 
the categorical variable would have implications for the types of statistical tests that could be conducted. While labelling of individual scores into three categories is somewhat arbitrary, use of the categorical variable may facilitate greater awareness of the differences between the three groups and generate additional research questions.

\section{Question 8: Attitude Toward the Development}

The formation of an attitude provides insights into subsequent behaviour and the potential for psychosocial health and wellbeing, or ill health and ill-being. Attitude towards a proposed development will reflect understandings of risk, and if such understandings of risk are considered high then the attitude is likely to be negative. A negative attitude may reflect levels of anguish and distress in some people. In our first paper (Authors 2018), we reviewed the associated mental health and well-being effects range from low-level ones, e.g. fear (Jacquet and Stedman 2014); anger (Taylor 2012; Ferrer and Klein 2015) (see Kubzandky and Kiwachi 2000, for a full review), and low-level anxiety (Luria et al. 2009; Jacquet and Stedman 2014) up to disabling anxiety disorders and conditions such as 'solastalgia' (Albrecht et al. 2007).

To measure attitude, one of the most widely accepted conceptual frameworks of an attitude is that it contains three elements which should all be measured: affective, cognitive and behavioural elements (Breckler 1984). A strongly held attitude is evident in all three areas and measuring all three provides greater reliability of the domain. The development of attitudinal items requires the incorporation of a clear judgement (for or against) in each item. Items would be measured on the same Likert scale as previously, and a mean score derived from respondents' answers to these items.

\section{Question 9: Linking Attitude Towards a Development with Symptoms of Poor Psychosocial Well-being}

While there is evidence of the link between attitude and psychological health and wellbeing, the relationship is not a given. There are many alternative explanations for psychological symptoms of ill health and ill-being which may be applicable. We used three items from the psychometrically reliable and valid DASS21 scale (Henry and Crawford 2005) to provide an indication only of possible psychological symptomatology that may or may not be related to the development proposal. The DASS21 is a 21item scale that measures the symptomatology of depression, anxiety and stress. One item from each of these three domains is included here to provide a crude marker of the possible levels of low mood, anxiety and stress in the community. No inferences about individual mental health and well-being status can be made from the use of these three items. These items were chosen because they were less gloomy sounding than other items, which is an important consideration for use with a non-clinical group. The items are non-specific to any urban or industrial development, and as such must be interpreted with caution. High symptomatology on one or more of these items may be closely associated with other events and occurrences in a person's life that are unrelated to the development. Nonetheless, we would expect that across a community that is potentially affected by a proposed development, mean scores on the sum of these three items will correlate positively with attitudes toward the development. If the association between attitude and indicators of psychosocial health and well-being is 
compelling, as evidenced by the strength of the association, it will provide confidence that the psychological symptomatology is at least in part related to the development proposal.

We opted not to word questionnaire items in way that would expressly connect the industrial facility with symptoms of psychological ill health or ill-being. Doing this would likely introduce unreliability in measurement because symptoms related to stress, anxiety and depression are not always attributable to one source, which would make it particularly problematic for individuals to accurately apportion any of their symptoms to the proposal.

It is possible that some participants will experience a heightened emotional state from talking and thinking about the industrial development. It is, therefore, important for researchers to provide a referral pathway to appropriate mental health support services for every participant should they need it. This type of information is typically included in a participant information statement about the study, given to every participant.

\section{Question 10: External Moderating Influences}

Researchers should insert the final list of external influences here decided upon by participants in Community Workshop 2. They can use the questions and scales proposed in the Workshop schedule in the Appendix.

\section{Survey Sampling and Data Collection}

Data would be collected from a sample of individuals from people living and working within the affected geographic area who may be potentially affected by the proposal. The size and whereabouts of this target population should be defined, and a sample size calculated that will be sufficient to answer the questions in the questionnaire.

Probability sampling methods would ideally be used to derive a representative sample so that inferences can be made to the target population. If this were not possible, say if there are no available sampling lists from which to derive a sampling frame, convenience sampling, i.e. sampling a selection of available individuals, is a preferable second-choice option. The disadvantage of convenience sampling is that results may not be generalisable to the target population. The demographics of the sample can be compared with what is known of the target population to assess the likelihood that a sample comes close (or not) to being representative.. Close approximation can provide greater confidence of the generalisability of the results, though does not guarantee it.

In situations where probability sampling is feasible, multi-stage stratified sampling is likely to be the most appropriate approach. The following example shows how it is comprised of stages. Stage 1 may involve sampling from a list of available administrative divisions (e.g. boroughs, suburbs, etc). Stage 2 may involve sampling streets from a list of all streets in the chosen administrative divisions. Stage 3 may involve sampling homes within sampled streets, and stage 4 may involve sampling individuals within sampled households.

Sampling quotas are often important for particular sub-population groups who are vulnerable to health and well-being effects or because they are an important vulnerable group within the local area and need to have their concerns heard. As well as proximity 
to the proposed project site, socio-demographic factors that may be used to stratify the sampling include age, language spoken, gender, socio-economic status, race/ethnicity, and parental status. If required, stratification will need to be built into the sampling plan to ensure adequate numbers are achieved which generally requires over sampling some groups and then making appropriate statistical adjustments during analysis.

\section{Quantitative Data Analysis}

The quantitative data collected from the survey would be analysed statistically. Since each construct in Fig. 1 is underpinned by several questions/items that have not previously been psychometrically tested for reliability and validity, it is important that the constructs (variables) in the psychosocial model prior undergo simple reliability and validity analysis prior to basing any decisions on the associations between construct variables. Internal consistency reliability (i.e. Cronbach's (1951) alpha coefficient) is used to assess levels of reliability for each construct and whether construct reliability would be improved by the omission of any items (refer to Alpha if item deleted information in statistical output for many statistical packages, which shows what the alpha coefficient would be if each item in the scale were omitted individually from the scale.). Satisfactory internal consistency reliability for research purposes is generally considered to be $>.70$ (Nunnally and Bernstein 1994). After deciding which items remain in each construct based on the simple reliability analysis described, each construct variable would be computed as a mean score of the items in each scale. Prior to conducting these analyses, it is essential that items are all worded in the same direction. Doing so would require recoding of all negatively worded items so that a higher score in all items reflects positive sentiment.

Basic construct validity analysis is conducted by correlating constructs with each other. Expected correlation coefficients between constructs for basic construct validity analysis is expected to be in the mid-range $(>.3-<.6)$ for variables that are linked by a direct path in Fig. 1. If these predictions are generally observed, then there would be some preliminary evidence of construct validity, though further work will be necessary to assess validity more comprehensively.

An analysis of the adequacy of the model overall, including all purported relationships between construct variables in the psychosocial conceptual model, would ideally be analysed using structural equation modelling (SEM) or through a series of regression analyses. SEM is preferred as it provides information about how well the data fits the model and also enables the modelling of latent variables such as experiential risk perceptions. However, running a series of regression analyses may be a more practical option for those unfamiliar with SEM and who do not have access to appropriate software. The use of regression analyses to test the paths in Fig. 1 is akin to path analysis (Loehlin 1992) which preceded the use of SEM techniques. Path analysis involves obtaining a correlation or regression coefficient for each of the specified pathways between variables. If variables are latent, such as experiential risk perceptions, a composite score would be computed from the mean score of the two variables deliberative risk perceptions + affective risk perceptions that lead to experiential risk perceptions. The purpose of the analyses would be to verify that the relationships purported in Fig. 1 are confirmed. If so, these factors could be relied upon as being relevant to community concerns. A description of how to use SEM or path analysis is 
beyond the scope here, but is summarised in Loehlin (1992) and multivariate statistical textbooks.

The external moderating factors that appear to be important in explaining understandings of risk as ascertained from discussions with the community at the stakeholder workshops may be tested for interaction effects using multiple linear regression or through SEM. Not all moderating factors should be tested otherwise an adjustment to the significance level is required to control for familywise error, which is the probability of making a Type 1 error. Having observed a significant interaction effect between an external factor and an understanding of risk, this information can be used to inform efforts to support population psychosocial well-being should the development proceed, .e.g. health promotion messages, risk communications strategies and community development interventions.

\section{Triangulation of Data}

The qualitative and quantitative data can be triangulated to cross-check how the survey responses on a large scale compare to the more in-depth workshop responses. Trends in response can be identified from the quantitative data, and from the qualitative data discrepancies, exceptions, anomalies and nuances in responses. Triangulation can provide validity to the picture of public understanding of risk that emerges from data collected, which can be written up using descriptive and statistical data points.

\section{Step 4: Hold a Feedback and Refinement Workshop}

The survey results are shared with community members at a final workshop, with the support of professional stakeholders. The community reviews the results and provide feedback, e.g. on disagreements they have, or exceptions, variations or anomalies that they are aware of. If mitigation measures or public communications have been developed using the qualitative data from the first workshop, then these can also be reviewed and validated.

\section{Implications of the Mixed Methodology}

We have conceived of psychosocial well-being as the influence of social factors on subjective mental health and well-being drawing on causal models proposed within social epidemiology, and the socio-ecological model of health. Whilst we are influenced by ideas of 'positive mental health and well-being', we have utilised 3 items from a 21-item clinical sub-scale for measuring symptoms of mental ill health and illbeing so as to provide an indication of symptoms that may arise from the psychological process described. This enables our results to be used by public health and well-being practitioners to indicate where the announcement of a proposed development may contribute to health problems that require the planning and health sectors to implement mitigation measures.

Our approach and methodology can be adopted by researchers guiding developers and planning officers. They can also be utilised by HIA practitioners collecting baseline 
data on the psychosocial effects of a development proposal in the pre-construction phase, and compiling evidence of how the health determinant, 'public understanding of risk' may change. Changes could occur from baseline to construction, to the operation or presence of a new development, and then again if/when there is a decommissioning phase. Our measurement proposal also allows understandings of risk to be categorised into 'high', 'medium' and 'low' in line with a risk management approach. This provides an easy-to-understand schema for those without well-being expertise, given the link between high risk understandings and symptoms of mental distress. The duration and frequency of any health effects can be monitored ongoingly if the community is re-surveyed at intervals throughout the lifecycle of the development, or after mitigation measures have been implemented, to assess their efficacy.

The psychosocial health and well-being effects of urban and industrial development are typically examined with reference to the baseline on common mental health disorders using routine population-level data (e.g. government or health sector statistics on anxiety or depression). This approach is problematic. In low- and middle-income countries, this information is typically not available. Also, this is an inadequate approach to community psychosocial health and well-being. The range of specific understandings of associated risk that local people develop cannot be established from routine data. Furthermore, statistics pertaining to individual mental health do not necessarily represent shared experiences at the group-level, nor do they reveal community-level drivers in response to risk experienced by groups (Greene et al. 2014). There is a risk of an 'ecological fallacy'; that is for example taking population-level measures of rates of mental health or even the relationships between causal factors for mental health symptoms and outcomes at a population level and erroneously inferring them to individuals in communities. The flip-side to ecological fallacy is that under certain circumstances (e.g. an unrepresentative sample), it may also be erroneous to collect data at an individual level and infer aggregated findings to the population in which the individuals belong. We have argued that it is possible to capture community experiences and sentiment about a proposed development, and the associated psychosocial well-being effects using our methodology.

Thirdly, it is not possible to directly attribute an increase in recorded cases of anxiety with heterogeneous understandings of a threat/risk. This is over simplistic and ignores the range of possible health and well-being effects. Anxiety, depression and stress are acute and chronic conditions in their own right. They are also health effects that result from the ways in which people understand risk. The way in which risk is understood and discussed is project, location, culture and time-specific. At all times there are individual and community-level factors at play. It is inevitable, in any community, that some people are more prone to anxiety and stress. There are also community discourses, i.e. collective understandings, that have a dynamic relationship with local factors.

For these reasons, we have argued that utilising a dedicated methodology that overcomes the limits of an etic well-being scale, by: 1) incorporating community experiences and perspectives into the design of the quantitative measurement tool using community-generated qualitative data; 2 ) triangulating the quantitative data with qualitative data on the community experiences, provides important evidence about psychosocial well-being. It provides both an accurate measurement of the influence of those experiences on aggregated individual psychosocial well-being, and rich description 
with which to contextualise those measurements. Our qualitative data collection strategy also amasses local people's judgements on the sources of risk: changes to the social and environmental determinants of health that may adversely affect them, that can be used to inform changes to development proposals or mitigation strategies to protect population subjective well-being.

\section{Conclusion}

The science of community well-being is an ever-evolving field in its own right, and in conjunction with important factors that influence well-being. We have shown how a key influence of urban and industrial development on well-being, public understanding of risk, may result in symptoms of poor psychosocial well-being, and outlined a partially-trialled mixed methodology to measure it. We argue that well-being research tools should incorporate the emic perspectives of those experiencing challenges to their well-being to ensure that measures of community well-being reflect shared experiences at the social level, and assist in the progression from measuring the mental ill health and ill-being of the pathologised individual to the well-being and resilience of many in a common social environment.

Author Contributions Cathy Baldwin:: conducted the background research, devised the conceptual framework and preliminary model, and wrote the first draft of the paper.

Ben Cave: Contributed to the text and provided comments on the draft.

Patrick Rawstorne: provided conceptual input to improve the model, produced Fig. 1, devised the quantitative methodology and wrote substantial text.

Compliance with Ethical Standards The authors declare that they have no conflict of interest. The research did not involve human or animal participation, therefore informed consent was not required.

Open Access This article is distributed under the terms of the Creative Commons Attribution 4.0 International License (http://creativecommons.org/licenses/by/4.0/), which permits unrestricted use, distribution, and reproduction in any medium, provided you give appropriate credit to the original author(s) and the source, provide a link to the Creative Commons license, and indicate if changes were made.

\section{References}

Albrecht, G., Sartore, G. M., Connor, L., Higginbotham, N., Freeman, S., Kelly, B., Stain, S., Tonna, A., \& Pollard, G. (2007). Solastalgia: the distress caused by environmental change. Australasian Psychiatry, 15(1), 41-55.

Anderson, J and Baldwin, C (2017) 'Building Wellbeing: Neighbourhood Flourishing and Approaches for Participatory Urban Design Intervention', in R. Phillips, C. Wong (eds.), Handbook of Community WellBeing Research, International Handbooks of Quality-of-Life, Springer Publications. https://doi. org/10.1007/978-94-024-0878-2_17

Baldwin, C and Rawstorne, P (2019). Public understanding of risk in health impact assessment: a psychosocial approach. Impact Assessment and Project Appraisal, 37(5), 382-396. https://doi.org/10.1080 /14615517.2018.1535952.

Baldwin, C. (2015). Assessing impacts on people's relationships to place and community in health impact assessment: An anthropological approach. Impact Assessment and Project Appraisal, 33(2), 154-159.

Baldwin, C, Vincent, P, Anderson, A and Rawstorne, P (under review) Measuring Well-being: Community Trial of the Neighbourhood Flourishing Framework for Pro-Social Individuals'. Submitted to International Journal of Community Wellbeing. 
Berkman, L. F., \& Kawachi, I. (Eds.). (2000). Social epidemiology. New York: Oxford University Press.

Birley, M. (2015). Social determinants of risk perception. London: Birley HIA. For IAIA15, Florence. Retrieved in 2016 from: http://conferences.iaia.org/2015/Final-Papers/Birley,\%20Martin\%20-\%20 Social\%20determinants\%20of\%20risk\%20perception.pdf. Accessed June 2016.

Breckler, S. J. (1984). Empirical validation of affect, behaviour, and cognition as distinct components of attitude. Journal of Personality and Social Psychology., 47(6), 1191-1205.

Brown, J. S. (2015). Promoting public mental health and well-being principles into practice. London and Philadelphia: Jessica Kingsley Publishers.

Creswell, J. W., Plano Clark, V. L., Guttman, M., \& Hanson, W. (2003). Advanced mixed methods research designs. In A. Tashakkori \& C. Teddlie (Eds.), Handbook of mixed methods in social \& behavioral research. Thousand Oaks: Sage.

Cronbach, L. J. (1951). Coefficient alpha and the internal structure of tests. Psychometrika, 16(3), $297-334$.

Dahlgren, G., \& Whitehead, M. (1991). Policies and strategies to promote social equity in health. Stockholm: Institute for Futures Studies: Stockholm.

Ferrer, R., \& Klein, W. M. (2015). Risk perceptions and health behavior. Current Opinion in Psychology, 5, 85-89.

Festinger, L. (1957). A theory of cognitive dissonance. London: Travistock Publications.

Glasgow Centre for Population Health. (2011). Asset-based approaches for health improvement: redressing the balance. Briefing paper 9, Concept Series. Glasgow: Glasgow Centre for Population Health. Retrieved in 2016 from: http://bit.ly/2h28swe Accessed June 2016.

Greene, G., Turley, R., Mann, M., Amlôt, R., Page, L., \& Palmer, S. (2014). Differing community responses to similar public health threats: A cross-disciplinary systematic literature review. Science of the Total Environment, 470, 59-767.

Hamilton, L. C. (1985). Concern about toxic wastes: Three demographic predictors. Sociological Perspectives, 28(4), 463-486.

Hanna, P., Vanclay, F., Langdon, E. J., \& Arts, J. (2016). The importance of cultural aspects in impact assessment and project development: Reflections from a case study of a hydroelectric dam in Brazil. Impact Assessment and Project Appraisal, 34(4), 306-318. https://doi.org/10.1080 /14615517.2016.1184501.

Harris, M. (1979). Cultural materialism: The struggle for a science of culture. New York: Vintage.

Henry, J. D., \& Crawford, J. R. (2005). The short-form version of the Depression Anxiety Stress Scale (DASS-21): Construct validity and normative data in a large non-clinical sample. British Journal of Clinical Psychology, 44(2), 227-239.

Higginbotham, M., Connor, L., Albrecht, G., Freeman, S., \& Agho, K. (2007). Validation of an environmental distress scale. EcoHealth, 3, 245-254.

Huppert, F. A., \& So, T. T. (2013). Flourishing across Europe: Application of a new conceptual framework for defining well-being. Social Indicators Research, 110, 837-861.

Huppert, F. A., \& Wittington, J. E. (2004). Positive mental health in individuals and populations. In F. A. Huppert \& B. N. Keverne (Eds.), The science of well-being (pp. 307-340). Oxford: Oxford University Press.

Jacquet, J. B., \& Stedman, R. C. (2014). The risk of social psychological disruption as an impact of energy development and environmental change. Journal of Environmental Planning and Management, 57(9), 1285-1304.

Keyes, C. L. M. (1998). Social well-being. Social Psychology Quarterly, 61, 121-140.

Keyes, C., Cicognani, E., Pirini, C., Joshanloo, M., Rostami, R., \& Nosratabadi. (2008). Social participation, sense of community and social well-being: Study on American, Italian and Iranian University Students. Social Indicators Research, 89, (1), 97-112.

Kubzandky, L. D., \& Kiwachi, I. (2000). Affective states and health. In L. F. Berkman \& I. Kawachi (Eds.), Social epidemiology (pp. 213-241). New York: Oxford University Press.

Larson, J. S. (1993). The measurement of social wellbeing. Social Indicators Research, 28, 285-289.

Laverack, G. (2001). An identification and interpretation of the organizational aspects of community empowerment. Community Development Journal, 36, 134-145.

Lazarus, R. (1999). Stress amd emotion: A new synthesis. New York: Springer Publishing.

Lima, M.L (2004). On the influence of risk perception on mental health: living near an incinerator. Journal of Environmental Psychology 24(1), 71-84.

Lima, M. L., \& Marques, S. (2005). Towards successful social impact assessment follow-up: A case study of psychosocial monitoring of a solid waste incinerator in the north of Portugal. Impact Assessment and Project Appraisal, 23(3), 227-233. 
Loehlin, J. C. (1992). Latent variable models: An introduction to factor, path, and structural analysis (2nd ed.). Hillsdale: Lawrence Erlbaum Associates.

Marcon, A., Nguyen, G., Rava, M., Braggion, M., Grassi, M., \& Zanolin, M. E. (2015). A score for measuring health risk perception in environmental surveys. Science of the Total Environment, 527-528, 270-278.

Martikainen, P., Bartley, M., \& Lahelma, E. (2002). Psychosocial determinants of health in social epidemiology. International Journal of Epidemiology, 31(6), 1091-1093.

McDowell, I and C. Newell, (1987). Measuring Health: A Guide to Rating Scales and Questionnaires. Toronto: Oxford University Press, pp 342.

Nunnally, J. C., \& Bernstein, I. H. (1994). Psychometric theory (3rd ed.). New York: McGraw-Hill.

Nutbeam, E., \& Harris, E. (2004). Chapter 3: Theories on change in communities and communal action for health. In Theory in a nutshell (2nd ed., pp. 10-24). Sydney: McGraw Hill.

Oatley, K., \& Johnson-Laird, P. N. (2013). Cognitive approaches to emotions. Trends in Cognitive Sciences, 1280, 1-7.

Parry, J., \& Wright, J. (2003). Community participation in health impact assessments: Intuitively appealing but practically difficult. Bulletin of the World Health Organization, 81(6), 388.

Pidgeon, N., Henwood, K., Parkhill, K., Venables, D., Simmons, P. (2008). Living with nuclear power in Britain: A mixed-methods study. UK: University of Cardiff and University of East Anglia. Retrieved on 25 May 2016 from: https://www.kent.ac.uk/scarr/SCARRNuclearReportPidgeonetalFINAL3.pdf; http://bit.ly/1TMAGdM. Accessed June 2016.

Ryan, R. M., \& Deci, E. L. (2001). On happiness and human potential: A review of research on hedonic and eudaimonic well-being. Annual Review of Psychology, 52, 141-166.

Scheier, M. F., Carver, C. S., \& Bridges, M. W. (1994). Distinguishing optimism from neuroticism (and trait anxiety, self-mastery, and self-esteem): A re-evaluation of the life orientation test. Journal of Personality and Social Psychology, 67(6), 1063-1078.

Schwarz, N., \& Clore, G. L. (1983). Mood, misattribution, and judgments of well-being: Informative and directive functions of affective states. Journal of Personality and Social Psychology, 45(3), 513-523.

Taylor, M. (2012). Review and evaluation of research literature on public nuclear risk perception and implications for communication strategies. Consultancy project for the Australian Uranium Association. Sydney: University of Western Sydney. Retrieved on 25 may 2016 from: http://www.minerals.org.au/file_ upload/files/reports/7.5D_Download_Report_Nuclear_Risk_Perception_Literature_Review_Report_ Final.pdf. Accessed June 2016.

Tennant, R., Hiller, L., Fishwick, R., Platt, S., Joseph, S., Weich, S., Parkinson, J., Secker, J., \& StewartBrown, S. (2007). The Warwick-Edinburgh Mental Well-being Scale (WEMWBS): Development and UK validation. Health and Quality of Life Outcomes Research Open Access, 5(63), 1-13. https://doi. org/10.1186/1477-7525-5-63.

Waterman, A. A. (1993). Two conceptions of happiness: Contrasts of personal expressiveness (eudaimonia) and hedonic enjoyment. Journal of Personality and Social Psychology 64(4), 678-691.

Wilkinson, R. and Marmot, M. (2003). Social determinants of health. The solid facts. $2^{\text {nd }}$ edition. Copenhagen: World Health Organization Europe regional office. Accessed on 10 $10^{\text {th }}$ October 2018 at: http://www.euro. who.int/_data/assets/pdf_file/0005/98438/e81384.pdf.

World Health Organisation. (2004). Promoting mental health; concepts emerging evidence and practice. Summary report. Geneva, World Health Organisation.

World Health Organization (1948/2006) Constitution of the World Health Organization. Basic Documents, Forty-fifth edition, Supplement, October 2006. Accessed on the 21dt November 2019 at: https:// www.who.int/governance/eb/who_constitution_en.pdf

Zajonc, R. B. (1980). Feeling and thinking: Preferences need no inferences. American Psychologist, 35, 151175 .

Publisher's Note Springer Nature remains neutral with regard to jurisdictional claims in published maps and institutional affiliations. 


\section{Affiliations}

\section{Cathy Baldwin ${ }^{1,2} \cdot$ Ben $^{\text {Cave }}{ }^{3} \cdot$ Patrick Rawstorne $^{4}$}

1 Institute of Health and Wellbeing, University of Glasgow, 1 Lilybank Gardens, Glasgow, Scotland G12 8RZ, UK

2 Institute for Social and Cultural Anthropology, University of Oxford, Oxford, UK

3 Ben Cave Associates Ltd, Leeds, UK, Ben Cave Associates Ltd, Gresham House, 5-7 St Pauls Street, Leeds LS1 2JG, UK

4 School of Public Health and Community Medicine, University of New South Wales, Australia, Samuels Building, F25, Samuel Terry Ave, Kensington, NSW 2033, Australia 